\title{
Cobalt Phthalocyanine - an Effective Catalyst of Hydrogen Production from Formic Acid
}

\author{
Evgeny V. Kudrik, ${ }^{@ 1}$ Sergei V. Makarov, ${ }^{@ 2}$ Elena S. Ageeva, and Ilia A. Dereven'kov \\ Ivanovo State University of Chemistry and Technology, Ivanovo, 153000, Russia \\ ${ }^{\circledR 1}$ Corresponding authorE-mail: ttos@isuct.ru \\ ${ }^{\circledR 2}$ Corresponding authorE-mail: makarov@isuct.ru
}

\begin{abstract}
Cobalt tetrasulfophthalocyanine, $\left.\mathrm{Co}^{\mathrm{II}}(\mathrm{TSP})^{4}\right)^{4}$, is shown to be an effective catalyst of decomposition of formic acid to hydrogen and carbon dioxide. The overall mechanism comprises the reduction of $\mathrm{Co}^{I I}(T S P c)^{4}$, followed by the reaction of intermediate complex $\mathrm{Co}^{\mathrm{I}}(\mathrm{TSP})^{{ }^{5-}}$ with proton.
\end{abstract}

Keywords: Tetrasulfophthalocyanine, cobalt complex, catalysis, formic acid decomposition, hydrogen production.

\section{Introduction}

The development of ways to capture and store renewable energy is one of the most important fields in chemistry. ${ }^{[1]} \mathrm{A}$ promising alternative to oil is hydrogen. ${ }^{[2]}$ It has the highest possible energy density of any hydrogen-based fuel. ${ }^{[2]}$ The transition to a Hydrogen Economy will require many technological advancements including findings of convenient hydrogen-producing systems. Formic acid is one of the promising precursors of hydrogen. It is well known that in the presence of platinum group metals or their salts formic acid decomposes to carbon dioxide and hydrogen under mild conditions. ${ }^{[3-5]}$ But decomposition of formic acid usually proceeds with low rates. ${ }^{\left[{ }^{[6]}\right.}$ This fact as well as the high cost of catalysts demand the studies of new catalytic systems. On the base of the data that $\mathrm{Co}^{\mathrm{I}}$ complexes are capable of reducing of proton, ${ }^{[7]}$ in this work we present the first data on catalytic properties of water-soluble Co tetrasulfophthalocyanine, $\mathrm{Co}^{\mathrm{II}}(\mathrm{TSP})^{4}$, in the reaction of formic acid decomposition.

\section{Experimental}

Materials. $\mathrm{Co}^{\mathrm{II}}(\mathrm{TSPC})^{4-}$ (1) was prepared and purified using literature method. ${ }^{[8]}$ All other chemicals were obtained from Aldrich and Acros Organics and used as received. TRIS, tris(hydroxymethyl)aminomethane, and MES, 2-(N-morpholino) ethanesulfonic acid, buffers were used to control $\mathrm{pH}$. Oxygen-free argon was used to deoxygenate the solutions.

Kinetic Measurements and Instrumentation. Mass spectra of reaction products were recorded on a JEOL Mstation 700 (EI, 70 $\mathrm{eV}$ ) spectrometer. Sodium formiate solution (0.1 M) in $20 \% \mathrm{HCOOH}$ was prepared under a dry argon atmosphere using standard Schlenk techniques. UV-vis spectra were recorded on a Cary 5 spectrophotometer under anaerobic conditions. Applied Photophysics SX 18MV stopped-flow spectrophotometer with an observation path length of $1.0 \mathrm{~cm}$ was used to follow the reactions. EPR spectra were recorded on Bruker ESP 300E spectrometer $(9.96 \mathrm{GHz})$ in $\mathrm{H}_{2} \mathrm{O}$ at $177 \mathrm{~K}$.

\section{Results and discussions}

It was shown earlier that complex $\mathbf{1}$ can be reduced in aqueous alkaline solutions by such a reductant as sodium dithionite ${ }^{[9]}$ The reaction is accompanied by the significant changes of UV-vis ${ }^{[9]}$ and EPR spectra. Thus, EPR spectrum of complex 1 contains singlet $(g=2.13)$ typical for low-spin $\mathrm{Co}^{\mathrm{II}}$ complex with unpaired electron localized in the $\mathrm{d}_{22}$ orbital. After addition of reductant this signal completely disappears that indicates the reduction of central atom. We found that subsequent addition of acid is accompanied by changing of solution colour from olive to blue, regeneration of EPR signal of less intensity and evolution of hydrogen the presence of which has been proved using mass-spectrometry. Since formiate is known to be an effective reductant a combining of both reductant (formiate) and oxidant (proton) in formic acid seems to be a very tempting task. Indeed, addition of complex 1 to $0.1 \mathrm{M}$ solution of sodium formiate in $20 \% \mathrm{HCOOH}$ is accompanied by evolution of gas. In mass-spectra of gas phase signals with $m / z=2\left(\mathrm{H}_{2}\right)$ and $44\left(\mathrm{CO}_{2}\right)$ were observed. These data indicate that complex $\mathbf{1}$ can be used as a catalyst of decomposition of formic acid. Supposing that a second step of catalytic process is a reaction between reduced form of cobalt tetrasulfophthalocyanine and proton we have studied kinetics of this reaction. Since reaction in strongly acidic solutions proceeds extremely fast we used weakly acidic solutions. For receiving of reduced Co complex $\left(10^{-6} \mathrm{M}\right)$ we used the reaction of $\mathrm{Co}^{\mathrm{II}}(\mathrm{TSP})^{4-}$ with an excess of sodium dithionite $\left(3 \cdot 10^{-6} \mathrm{M}\right)$ under anaerobic conditions. Rate constants of reaction between $\mathrm{Co}^{\mathrm{I}}(\mathrm{TSP})^{5-}$ and proton were determined from the decrease in the absorbance maximum at $450 \mathrm{~nm}$ corresponding to $\mathrm{Co}^{\mathrm{I}}$ complex..$^{[9]}$ The order with respect to proton (1.7) was found from the van't Hoff plot (Figure 1).

This value, i.e. between 1 and 2, as well as a rate dependence on $\left[\mathrm{H}^{+}\right]$(Figure 2) suggest a combination of two reaction paths - of the first and second-order, respectively:

$$
\begin{array}{ll}
\text { 1. } & \mathrm{Co}^{\mathrm{I}}+\mathrm{H}^{+} \stackrel{k_{1}}{\longrightarrow} \mathrm{Co}^{\mathrm{III}}-\mathrm{H} \\
& \mathrm{Co}^{\mathrm{III}}-\mathrm{H}+\mathrm{Co}^{\mathrm{III}}-\mathrm{H} \stackrel{k_{2}}{\longrightarrow} 2 \mathrm{Co}^{\mathrm{II}}+\mathrm{H}_{2} \\
\text { 2. } & \mathrm{Co}^{\mathrm{I}}+\mathrm{H}^{+} \stackrel{k_{1}}{\longrightarrow} \mathrm{Co}^{\mathrm{III}}-\mathrm{H} \\
& \mathrm{Co}^{\mathrm{III}}-\mathrm{H}+\mathrm{H}^{+} \stackrel{k_{3}}{\longrightarrow} \mathrm{Co}^{\mathrm{II}}+\mathrm{H}_{2}
\end{array}
$$




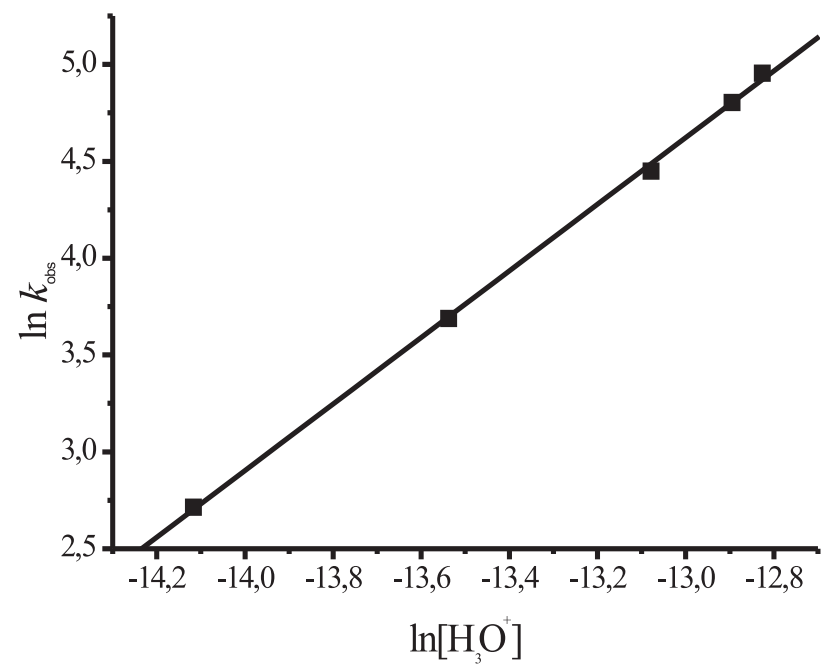

Figure 1. van't Hoff plot for the reaction between $\mathrm{Co}^{\mathrm{I}}(\mathrm{TSP})^{5-}$ and $\mathrm{H}^{+}$at $298 \mathrm{~K}$.

The suggested mechanism is similar to the mechanism of electrocatalytic reduction of proton in the presence of cobalt complexes with other aza-ligands. ${ }^{[10]}$

Since reaction rate does not depend on the nature of the used acid (HA) one can conclude that $k_{\text {obs }}$ should be expressed by Equation 1:

$$
k_{o b s}=k_{1} k_{2}\left[\mathrm{H}^{+}\right]+k_{1} k_{3}\left[\mathrm{H}^{+}\right]^{2}
$$

At low $\mathrm{pH}$ Equation 1 can be simplified to Equation 2:

$$
k_{o b s} \approx k_{1} k_{3}\left[\mathrm{H}^{+}\right]^{2}
$$

According to Equation 1 and data of Figure 2 the values of $k_{\mathrm{I}}$ and $k_{\mathrm{II}}\left(k_{\mathrm{I}}=k_{1} \cdot k_{2}=(6.9 \pm 2.0) \cdot 10^{6} \mathrm{~s}^{-1}\right.$, $\left.k_{\mathrm{II}}=k_{1} \cdot k_{3}=(1.7 \pm 0.8) \cdot 10^{13} \mathrm{M}^{-1} \cdot \mathrm{s}^{-1}\right)$ were determined.

It was found that the reaction rate very weakly depends on temperature (the activation energy determined from the Arrehnius plot is only $2 \mathrm{~kJ} / \mathrm{mol}$ ). Apparently, this is due to reaction rate's limitation by diffusion as could also be seen from the high numerical value of the second-order kinetic constant. Diffusion-limited reactions are characteristic for proton-transfer reactions in solutions provided that the other reaction steps are fast. The received data indicate the high efficiency of cobalt tetrasulfophthalocyanine as a catalyst of formic acid decomposition.

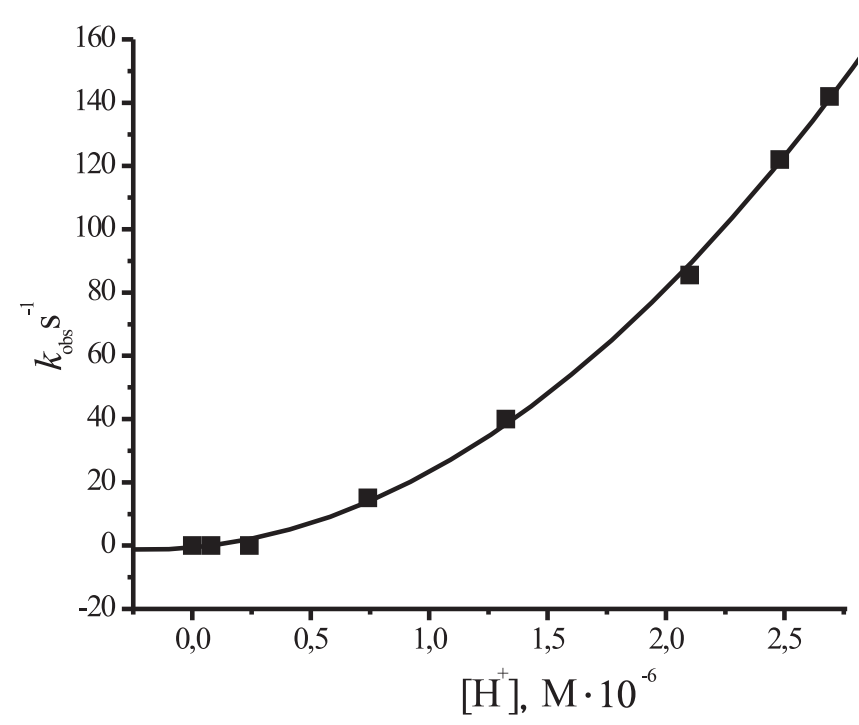

Figure 2. The dependence of $k_{\text {obs }}$ on $\left[\mathrm{H}^{+}\right]$at $298 \mathrm{~K}$.

\section{Conclusions}

This study has shown that cobalt tetrasulfophthalocyanine is an effective catalyst for hydrogen production from formic acid. The catalytic cycle includes the reversible reaction $\mathrm{Co}^{\mathrm{II}} \leftrightarrow \mathrm{Co}^{\mathrm{I}}$ accompanied by decomposition of formic acid to hydrogen and carbon dioxide.

\section{References}

1. Armstrong F.A., Belsey N.A., Cracknell J.A., Goldet G., Parkin A., Reisner E., Vincent K.A., Wait A.F. Chem. Soc. Rev. 2009, 38, 36-51.

2. Graetz J. Chem. Soc. Rev. 2009, 38, 73-82.

3. Yoshida T., Ueda Y., Otsuka S. J. Am. Chem. Soc. 1978, 100, 3941-3942.

4. Coffey R. S. Chem. Commun. 1967, 923-924.

5. Strauss S.H., Whitmire K.H., Shriver D.F. J. Organomet. Chem. 1979, 174, C59-C62.

6. Fellay C., Dyson P. J., Laurenczy G. Angew. Chem. Int. Ed. 2008, 47, 3966-3968.

7. Tait A.M., Hoffman M.Z., Morton Z., Hayon E. J. Am. Chem. Soc. 1976, 98, 83-93.

8. Weber J.H., Busch D. H. Inorg.Chem. 1965, 1, 469-471.

9. Kudrik E.V., Makarov S.V., Zahl A., van Eldik R. Inorg. Chem. 2003, 42, 618-624.

10. Hu H., Brunschwig B.S., Peters J. J. Am. Chem. Soc. 2007, 129, 8988-8998. 